PROCEEDINGS OF THE

AMERICAN MATHEMATICAL SOCIETY

Volume 137, Number 5, May 2009, Pages 1601-1611

S 0002-9939(08)09739-6

Article electronically published on November 20, 2008

\title{
THE GAUSS-BONNET FORMULA OF POLYTOPAL MANIFOLDS AND THE CHARACTERIZATION OF EMBEDDED GRAPHS WITH NONNEGATIVE CURVATURE
}

\author{
BEIFANG CHEN \\ (Communicated by Jon G. Wolfson)
}

\begin{abstract}
Let $M$ be a connected $d$-manifold without boundary obtained from a (possibly infinite) collection $\mathcal{P}$ of polytopes of $\mathbb{R}^{d}$ by identifying them along isometric facets. Let $V(M)$ be the set of vertices of $M$. For each $v \in V(M)$, define the discrete Gaussian curvature $\kappa_{M}(v)$ as the normal angle-sum with sign, extended over all polytopes having $v$ as a vertex. Our main result is as follows: If the absolute total curvature $\sum_{v \in V(M)}\left|\kappa_{M}(v)\right|$ is finite, then the limiting curvature $\kappa_{M}(p)$ for every end $p \in \operatorname{End} M$ can be well-defined and the Gauss-Bonnet formula holds:

$$
\sum_{v \in V(M) \cup \text { End } M} \kappa_{M}(v)=\chi(M) .
$$

In particular, if $G$ is a (possibly infinite) graph embedded in a 2-manifold $M$ without boundary such that every face has at least 3 sides, and if the combinatorial curvature $\Phi_{G}(v) \geq 0$ for all $v \in V(G)$, then the number of vertices with nonvanishing curvature is finite. Furthermore, if $G$ is finite, then $M$ has four choices: sphere, torus, projective plane, and Klein bottle. If $G$ is infinite, then $M$ has three choices: cylinder without boundary, plane, and projective plane minus one point.
\end{abstract}

\section{Polytopal manifolds}

Let $N$ be a compact $d$-dimensional manifold without boundary; as usual we call it a closed $d$-manifold. Let $M$ be a $d$-dimensional submanifold of $N$ such that $N-M$ is a finite set. We call $M$ a d-manifold with finite number of ends and write End $M:=N-M$; each element of End $M$ is called an end of $M$. By an open convex $k$-cell (or just a $k$-cell) of $M$ we mean a subset $\sigma \subseteq M$ together with an embedding $\phi: \bar{\sigma} \rightarrow \mathbb{R}^{d}$, where $\bar{\sigma}$ is the closure of $\sigma$ in $N$, such that

(C1) $\phi(\bar{\sigma})$ is a $k$-dimensional compact convex set of $\mathbb{R}^{d}$, and

$(\mathrm{C} 2) \phi(\sigma)$ is the relative interior of $\phi(\bar{\sigma})$;

Received by the editors March 2, 2007, and, in revised form, February 15, 2008, and, July 6, 2008.

2000 Mathematics Subject Classification. Primary 05C10, 52B70; Secondary 05C75, 57M15, 57N05, 57P99.

Key words and phrases. Discrete curvature, combinatorial curvature, Gauss-Bonnet formula, Euler relation, infinite graph, embedded graph, nonnegative curvature, finiteness theorem.

The author was supported in part by the RGC Competitive Earmarked Research Grants 600703 and 600506.

(C)2008 American Mathematical Society Reverts to public domain 28 years from publication 
we call $\bar{\sigma}$ a closed $k$-cell and $\partial \sigma:=\bar{\sigma}-\sigma$ the boundary of $\sigma$. A cell $\sigma$ is called a face of a cell $\tau$, written $\sigma \leq \tau$, if either $\sigma=\tau$ or $\sigma \subset \partial \tau$. A face $\sigma$ of $\tau$ is called a facet if $\operatorname{dim} \sigma=\operatorname{dim} \tau-1$. A convex cell complex on $M$ is a (possibly infinite) collection $\mathcal{C}(M)$ of relatively open convex cells of $M$ such that

(CC1) If $\sigma, \tau \in \mathcal{C}(M)$ and $\sigma \cap \bar{\tau} \neq \emptyset$, then $\bar{\sigma} \subseteq \bar{\tau}$.

(CC2) $M=\bigsqcup_{\sigma \in \mathcal{C}(M)} \sigma$.

(CC3) For each $\sigma \in \mathcal{C}(M)$, the collection $\{\tau \in \mathcal{C}(M) \mid \sigma \leq \tau\}$ is finite.

A subspace $S$ of $M$ is said to be cellular if there is a subcollection $\mathcal{C}(S)$ of $\mathcal{C}(M)$ such that $S=\bigcup_{\sigma \in \mathcal{C}(S)} \sigma$.

By a polytopal structure on $M$ we mean a cellular complex $\mathcal{C}(M)$ satisfying the following conditions:

(PM1) For each $d$-cell $\sigma$ there exists an embedding $\phi_{\sigma}: \bar{\sigma} \rightarrow \mathbb{R}^{d}$ such that the image $\phi_{\sigma}(\sigma)$ is an open $d$-polytope. We call $\left(\sigma, \phi_{\sigma}\right)$ a polytopal chart and $\sigma$ an open d-polytope of $M$.

(PM2) If $\sigma$ and $\tau$ are $d$-cells such that $\bar{\sigma} \cap \bar{\tau} \neq \emptyset$, then $\phi_{\tau} \circ \phi_{\sigma}^{-1}: \phi_{\sigma}(\bar{\sigma} \cap \bar{\tau}) \rightarrow \phi_{\tau}(\bar{\sigma} \cap \bar{\tau})$ can be extended to an isometry of $\mathbb{R}^{d}$.

We call a manifold with a polytopal structure a polytopal manifold and call 0-cells vertices, 1-cells open segments, and 2-cells open convex polygons. We denote by $V(M)$ the set of vertices. A subspace $X \subseteq M$ is said to be a polyhedron if, for each polytopal chart $\left(\sigma, \phi_{\sigma}\right)$, the image $\phi_{\sigma}(X \cap \bar{\sigma})$ is a disjoint union of finitely many relatively open polytopes of $\phi_{\sigma}(\bar{\sigma})$; i.e., $\phi_{\sigma}(X \cap \bar{\sigma})$ is a polyhedron of $\mathbb{R}^{d}$ in the sense of [6]. A polyhedron $X$ of $M$ is said to be bounded if the closure $\bar{X}$ does not contain any end. If $X$ is bounded, then $X$ intersects only a finite number of cells in $\mathcal{C}(M)$.

We lack references for polytopal manifolds of dimensions $\geq 3$. For dimension 2 , the book of Aleksandrov and Zalgaller [1] is a good source for polygonal surfaces. For the motivation of Gaussian curvature below, we refer to the work of Allendoerfer and Weil [2], Banchoff [3], Cheeger, Müller, and Schrader [5], McMullen [13, and the present author [6]. For the motivation of combinatorial curvature, we refer to Gromov [10, Higuchi [11, and Ishida [12. For the application of the Gaussian curvature and the combinatorial curvature on surfaces, we refer to the recent work of DeVos and Mohar [8] and the author's joint work with G. Chen [7].

Let $M$ be a polytopal $d$-manifold. A geodesic segment of $M$ is an embedding $g: I \rightarrow M$, where $I$ is a closed interval of $\mathbb{R}$, such that $g(I)$ is contained in a closed $d$-cell $\bar{\sigma}$ and $\phi_{\sigma}(g(I))$ is a segment in the polytope $\phi_{\sigma}(\bar{\sigma})$. The length of a geodesic segment $g$, denoted $\ell(g)$, is the length of the segment $\phi_{\sigma}(g(I))$ in $\mathbb{R}^{d}$. A geodesic walk is a continuous map $\gamma:[a, b] \rightarrow M$ such that $[a, b]$ can be partitioned into $a=a_{0}<a_{1}<\cdots<a_{m}=b$ so that the restrictions $\left.\gamma\right|_{\left[a_{i-1}, a_{i}\right]}$ are geodesic segments. The length of a geodesic walk $\gamma$ is defined to be

$$
\ell(\gamma):=\sum_{i=1}^{m} \ell\left(\left.\gamma\right|_{\left[a_{i-1}, a_{i}\right]}\right) .
$$

A geodesic path is a geodesic walk $\gamma:[a, b] \rightarrow M$ such that the restriction $\left.\gamma\right|_{(a, b)}$ to the open interval $(a, b)$ is injective. A geodesic walk (path) $\gamma:[a, b] \rightarrow M$ is said to be closed if $\gamma(a)=\gamma(b)$. There is a canonical metric $d$ on a polytopal manifold $M$, whose distance $d(x, y)$ between two points $x, y \in M$ is the infimum of the lengths of all geodesic paths from $x$ to $y$. 
Let $B^{d}$ denote the closed unit ball of $\mathbb{R}^{d}$ centered at the origin $o$. Let $C$ be a convex cone of $\mathbb{R}^{d}$ whose cone point is the origin $o$, where $\operatorname{dim} C$ may be lower than $d$. The normal cone of $C$ is the closed convex cone

$$
C^{*}:=\left\{v \in \mathbb{R}^{d} \mid\langle u, v\rangle \leq 0 \text { for all } u \in C\right\} .
$$

Let $P$ be a polyhedron of $\mathbb{R}^{d}$. For each $p \in \bar{P}$, the tangent cone of $P$ at $p$ is the polyhedral cone

$$
T_{p}(P):=\left\{v \in \mathbb{R}^{d} \mid \exists \varepsilon>0 \text { s.t. } p+t v \in P \forall 0<t<\varepsilon\right\} .
$$

Let $T_{p}(P)$ be written as a disjoint union of finitely many relatively open convex cones $C_{i}$. The Gaussian curvature of $P$ at $p$ is defined in [6] as

$$
\kappa_{P}(p):=\sum_{i}(-1)^{\operatorname{dim} C_{i}} \frac{\operatorname{vol}\left(C_{i}^{*} \cap B^{d}\right)}{\operatorname{vol}\left(B^{d}\right)},
$$

where vol is the Lebesgue measure on the Euclidean space $\mathbb{R}^{d}$. When $p \notin \bar{P}$, we take the convention $\kappa_{P}(p)=0$. It is known by Groemer's extension theorem [9] (see Lemma 2.1 and Lemma 4.1 in [6]) that $\kappa_{P}(p)$ is independent of how $T_{p}(P)$ is written as a disjoint union of finitely many relatively open convex cones. The curvature function $\kappa_{P}(p)$ is additive in the sense that if $Q$ is a polyhedron of $\mathbb{R}^{d}$, then

$$
\kappa_{P \cup Q}(p)=\kappa_{P}(p)+\kappa_{Q}(p)-\kappa_{P \cap Q}(p), \quad p \in \mathbb{R}^{d} .
$$

It is clear that $\kappa_{P}(p)$ is invariant under isometries of $\mathbb{R}^{d}$.

Let $X$ be a polyhedron of $M$. We denote by $\mathcal{C}(X)$ the collection of cells $\sigma \in \mathcal{C}(M)$ such that $X \cap \sigma \neq \emptyset$. For each cell $\sigma \in \mathcal{C}(M)$, let $\mathcal{C}_{d}(M, \sigma)$ denote the collection of all $d$-cells $\tau$ such that $\sigma \leq \tau$. We define the Gaussian curvature of $X$ at $x \in M$ to be

$$
\kappa_{X}(x):=\sum_{\sigma \in \mathcal{C}(X), x \in \bar{\sigma}} \frac{1}{\#\left(\mathcal{C}_{d}(M, \sigma)\right)} \sum_{\tau \in \mathcal{C}_{d}(M, \sigma)} \kappa_{\phi_{\tau}(X \cap \sigma)}\left(\phi_{\tau}(x)\right) .
$$

So the Gaussian curvature of $X$ is a well-defined function $\kappa_{X}: M \rightarrow \mathbb{R}$. Note that for each cell $\sigma \in \mathcal{C}(M), \kappa_{X}(x)$ is nonzero at only finitely many points of $\bar{\sigma}$; and $\kappa_{X}(x)=0$ for $x \in M-\bar{X}$. The curvature function $\kappa_{X}(x)$ is clearly also additive.

Similar to the combinatorial curvature of [10, 11, 12 for embedded graphs, the combinatorial curvature for a cellular subspace $S$ of $M$ can be defined as a function $\Phi_{S}: V(M) \rightarrow \mathbb{R}$, given by

$$
\Phi_{S}(v):=\sum_{\sigma \in \mathcal{C}(S), v \in \bar{\sigma}} \frac{(-1)^{\operatorname{dim} \sigma}}{\#(V(\bar{\sigma}))} .
$$

Notice that for each cell $\sigma \in \mathcal{C}(M)$,

$$
\sum_{x \in V(\bar{\sigma})} \kappa_{\sigma}(x)=\sum_{x \in V(\bar{\sigma})} \Phi_{\sigma}(x)=(-1)^{\operatorname{dim} \sigma} .
$$

It follows that for any finite cellular subspace $S$ of $M$, we have the Gauss-Bonnet formula (for finite cellular subspaces):

$$
\sum_{v \in V(\bar{S})} \kappa_{S}(v)=\sum_{v \in V(\bar{S})} \Phi_{S}(v)=\chi(S) .
$$


More generally, for any bounded polyhedron $X$ of $M$, there is also the Gauss-Bonnet formula (for bounded polyhedra):

$$
\sum_{x \in \bar{X}} \kappa_{X}(x)=\chi(X)
$$

In the next section we prove the Gauss-Bonnet formulas for polyhedra that may intersect infinitely many cells in $\mathcal{C}(M)$. The crucial part is to define the Gaussian curvature correctly at each end of the polytopal manifold $M$. In section 3 we show that the finiteness of the absolute total curvature implies the finiteness of the number of vertices of nonvanishing curvature by the Gauss-Bonnet formulas. In the special case of dimension 2, we characterize regular polygonal surfaces having nonnegative curvature everywhere by the Gauss-Bonnet formula for surfaces with finite ends, and solve a conjecture made previously by the author. The variety of such graphs is much richer than that of graphs with positive curvature everywhere.

\section{The Gauss-Bonnet formula}

Let $M$ be a polytopal $d$-manifold with a finite number of ends in a closed $d$ manifold $N$; i.e., End $M=N-M$ is a finite set. To have a Gauss-Bonnet formula for $M$, we need to define the curvature at each end of $M$. Let $p$ be an end of $M$. Let $U_{n}(p)$ be a sequence of closed neighborhoods of $p$ in $N$, satisfying the following conditions:

(S1) $\partial U_{n}(p)$ are cellular subspaces;

(S2) $\left(U_{n}(p), \partial U_{n}(p)\right)$ are homeomorphic to $\left(B^{d}, \partial B^{d}\right)$;

(S3) $U_{n}(p) \subset \operatorname{int} U_{n-1}(p)$, and $\bigcap_{n=1}^{\infty} U_{n}(p)=\{p\}$.

Each of $U_{n}(p)$ is called a cellular neighborhood of $p$ in $N$. Then $M_{n}:=N-U_{n}$ are polyhedra of $M$. We define the Gaussian curvature of $M$ at $p$ to be the limit (if it exists)

$$
\kappa_{M}(p):=\lim _{n \rightarrow \infty} \sum_{x \in V\left(\partial U_{n}(p)\right)} \kappa_{M_{n}}(x) .
$$

Theorem 2.1 (The Gauss-Bonnet formula). Let $M$ be a polytopal d-manifold with a finite number of ends in a closed d-manifold $N$ : End $M=N-M$. If the absolute total curvature $|\kappa|(M)=\sum_{v \in V(M)}\left|\kappa_{M}(v)\right|$ is finite, then the limiting curvature $\kappa_{M}(p)$ exists for every end $p \in$ End $M$ and the Gauss-Bonnet formula holds:

$$
\sum_{v \in V(N)} \kappa_{M}(v)=\chi(M),
$$

where $V(N)=V(M) \cup$ End $M$ is the closure of $V(M)$ in $N$ and $\chi(M):=\chi(N)$ \# $($ End $M)$ is the Euler characteristic of $M$.

The Gauss-Bonnet formula (2.1) may be stated for tame polyhedra of $M$. A polyhedron $X$ of $M$ is said to be tame provided that for each end $p \in \bar{X} \cap$ End $M$, there exists a sequence of closed cellular neighborhoods $U_{n}$ of $p$ in $N$ such that:

(T1) $U_{n}$ satisfy the conditions (S1)-(S3),

(T2) $X \cap U_{n} \simeq\left(X \cap \partial U_{n}\right) \times[0,1)$.

For example, the manifold $M$ is a tame polyhedron of itself. So Theorem 2.1 is a special case of the following Theorem 2.3. Nontame polyhedra do exist and can be easily demonstrated. 
We wish to define the limiting curvature $\kappa_{X}(p)$ of a tame polyhedron $X$ at each of its ends. Fix an end $p \in \bar{X} \cap$ End $M$; let $U_{n}(p)$ be a closed cellular neighborhood of $p$ in $N$ satisfying (T1) and (T2). Then $X_{n}:=X-U_{n}(p)$ is a polyhedron of $M$. We define the Gaussian curvature of $X$ at $p$ to be the limit (if it exists)

$$
\kappa_{X}(p):=\lim _{n \rightarrow \infty} \sum_{x \in \partial U_{n}(p)} \kappa_{X_{n}}(x) .
$$

Note that $\kappa_{X_{n}}(x)$ are nonzero at only a finite number of points of $\partial U_{n}(p)$.

Lemma 2.2. Let $p \in \bar{X} \cap$ End $M$ be an end of a tame polyhedron $X$. If there exists a cellular neighborhood $U$ of $p$ in $N$ such that $\sum_{x \in U, x \neq p}\left|\kappa_{X}(x)\right|<\infty$, then the limiting curvature $\kappa_{X}(p)$ exists.

Proof. Note that for any cell $\sigma \in \mathcal{C}(M)$, the curvature $\kappa_{X}(x)$ is zero on $\bar{\sigma}$ except for a finite number of points. The summation is understood that all finite sums are bounded above by a fixed number $K$. Let $U_{n}$ and $W_{n}$ be sequences of closed cellular neighborhoods of $p$ in $N$, satisfying (T1) and (T2). Write $X_{n}=X \cap\left(N-U_{n}\right)$, $Y_{n}=X \cap\left(N-W_{n}\right)$, and define the sequences:

$$
a_{n}=\sum_{x \in \partial U_{n}} \kappa_{X_{n}}(x), \quad b_{n}=\sum_{x \in \partial W_{n}} \kappa_{Y_{n}}(x) .
$$

These sequences are well-defined because $\kappa_{X_{n}}$ and $\kappa_{Y_{n}}$ are nonzero at only a finite number of points of $\partial U_{n}$ and $\partial W_{n}$, respectively. Consider positive integers $m, n$ such that $W_{n} \subset \operatorname{int} U_{m}$. Set $X_{m, n}=X \cap\left(U_{m}-W_{n}\right)$ and $X_{m, n}^{\prime}=\bar{X} \cap\left(U_{m}-W_{n}\right)$. Then $X_{m, n}$ is a bounded polyhedron of $M$. Note that $X_{m, n}$ is homeomorphic to $\left(X \cap \partial U_{m}\right) \times[0,1)$. Since $\chi([0,1))=0$, the Gauss-Bonnet formula (for bounded polyhedra) implies

$$
\sum_{x \in \bar{X}_{m, n}} \kappa_{X_{m, n}}(x)=\chi\left(X_{m, n}\right)=\chi\left(X \cap \partial U_{m}\right) \chi([0,1))=0 .
$$

Notice that $\kappa_{Y_{n}}(x)=\kappa_{X_{m, n}}(x)$ for $x \in \partial W_{n}$ and $\partial W_{n}=\bar{X}_{m, n}-X_{m, n}^{\prime}$. The difference $b_{n}-a_{m}$ can be written as

$$
\begin{aligned}
b_{n}-a_{m} & =\sum_{x \in \partial W_{n}} \kappa_{X_{m, n}}(x)-\sum_{x \in \partial U_{m}} \kappa_{X_{m}}(x) \\
& =\sum_{x \in \bar{X}_{m, n}-X_{m, n}^{\prime}} \kappa_{X_{m, n}}(x)-\sum_{x \in \partial U_{m}} \kappa_{X_{m}}(x) \\
& =-\sum_{x \in X_{m, n}^{\prime}} \kappa_{X_{m, n}}(x)-\sum_{x \in \partial U_{m}} \kappa_{X_{m}}(x) .
\end{aligned}
$$

Now for $x \in X_{m, n}^{\prime}$ but $x \notin \partial U_{m}$, we have $\kappa_{X_{m, n}}(x)=\kappa_{X}(x)$; while for $x \in \partial U_{m}$, we have $\kappa_{X_{m, n}}(x)+\kappa_{X_{m}}(x)=\kappa_{X}(x)$. It turns out that

$$
b_{n}-a_{m}=-\sum_{x \in X_{m, n}^{\prime}} \kappa_{X}(x)
$$

Since $\sum_{x \in U_{m}, x \neq p}\left|\kappa_{X}(x)\right|<\infty$ for large enough $m$, we thus have

$$
\left|b_{n}-a_{m}\right| \leq \sum_{x \in U_{m}, x \neq p}\left|\kappa_{X}(x)\right| \rightarrow 0(m \rightarrow \infty) .
$$


Taking $U_{n}=W_{n}$, one sees that $a_{n}$ is a Cauchy sequence. Of course its limit exists, and such a limit is independent of the chosen sequences of the cellular neighborhoods.

Theorem 2.3 (The Gauss-Bonnet formula for polyhedra). Let $X$ be a tame polyhedron of $M$. If the absolute total curvature $|\kappa|(X)=\sum_{x \in \bar{X} \cap M}\left|\kappa_{X}(x)\right|$ is finite, then the limiting curvature $\kappa_{X}(p)$ exists for each end $p \in \bar{X} \cap$ End $M$, and we have the Gauss-Bonnet formula

$$
\sum_{x \in \bar{X}} \kappa_{X}(x)=\sum_{x \in \bar{X} \cap M} \kappa_{X}(x)+\sum_{p \in \bar{X} \cap \operatorname{End} M} \kappa_{X}(p)=\chi(X) .
$$

Proof. For each $p \in$ End $M$, let $U_{n}(p)$ be a sequence of closed cellular neighborhoods of $p$ in the manifold $N$ such that (T1)-(T2) are satisfied. We may further assume that $U_{n}(p)$ are disjoint for all $p \in$ End $M$. Then

$$
M_{n}=M-\bigcup_{p \in \operatorname{End} M} U_{n}(p)
$$

is a finite cellular subspace of $M$. We define $X_{n}=X \cap M_{n}$. Let $M_{n}(p)=M-U_{n}(p)$ and $X_{n}(p)=X \cap M_{n}(p)$. Then $X_{n}=\bigcap_{p \in \text { End } M} X_{n}(p)$. Applying the Gauss-Bonnet formula (for finite cellular subspaces) to $X_{n}$, we have

$$
\begin{aligned}
\chi\left(X_{n}\right) & =\sum_{x \in \bar{X}_{n}} \kappa_{X_{n}}(x)=\sum_{x \in \bar{M}_{n}} \kappa_{X_{n}}(x) \\
& =\sum_{x \in M_{n}} \kappa_{X_{n}}(x)+\sum_{p \in \operatorname{End}} \sum_{M \in \partial U_{n}(p)} \kappa_{X_{n}}(x) .
\end{aligned}
$$

Note that $\kappa_{X_{n}}(x)=\kappa_{X}(x)$ for $x \in V\left(M_{n}\right)$ and $X_{n}$ is homeomorphic to $X$. Then $\chi\left(X_{n}\right)=\chi(X)$. Let $n$ tend to infinity and make use of the definition of $\kappa_{X}(p)$ for $p \in$ End $M$. We obtain

$$
\chi(X)=\sum_{x \in M} \kappa_{X}(x)+\sum_{p \in \operatorname{End} M} \kappa_{X}(p)=\sum_{x \in \bar{X}} \kappa_{X}(x) .
$$

Let $S$ be a cellular subspace of $M$. Then $\kappa_{S}(x)$ are zero except at the vertices of $\bar{S} \cap M$. In particular, for the polytopal manifold $M, \kappa_{M}(x)$ is zero except on the vertex set $V(M)$.

Corollary 2.4 (The Gauss-Bonnet formula for cellular subspaces). Let $S$ be a tame cellular subspace of $M$. If the absolute total curvature

$$
|\kappa|(S)=\sum_{x \in V(\bar{S} \cap M)}\left|\kappa_{S}(x)\right|
$$

is finite, then the limiting curvature $\kappa_{S}(p)$ exists for each end $p \in$ End $M$, and we have the Gauss-Bonnet formula

$$
\sum_{x \in V(\bar{S} \cap M)} \kappa_{S}(x)+\sum_{p \in \bar{S} \cap \operatorname{End} M} \kappa_{S}(p)=\chi(S) .
$$




\section{The Characterization of EMBEDDED GRAPHS With NONNEGATIVE COMBINATORIAL CURVATURE}

In this section we assume that $M$ is a polytopal 2-manifold with a finite number of ends in a closed 2-manifold $N$; such an $M$ is usually called a polygonal surface. The 1-skeleton forms a graph $G$ embedded in $M$. We denote by $V(G)$ the set of vertices, and by $E(G)$ the set of edges of the graph $G$. Let

$$
\nu(M):=\inf \{d(u, v) \mid u, v \in V(M), u \neq v\} .
$$

DeVos and Mohar [8] obtained the following Gauss-Bonnet inequality (3.1), which solves a conjecture of Higuchi [1]. Higuchi's conjecture can be thought of as a combinatorial analogue of Myers' theorem [4, 14] on Riemannian manifolds. Other related results are the work of Stone [15] and Woess [16].

Theorem 3.1 (DeVos and Mohar). Let $M$ be a polygonal surface with a finite number of ends. If $\nu(M)>0$ and $\sum_{v \in V(M), \kappa_{M}(v)<0} \kappa_{M}(v)$ converges, then

$$
\sum_{v \in V(M)} \kappa_{M}(v) \leq \chi(M) .
$$

Let $G$ be a (possibly infinite) graph embedded in a 2-manifold with a finite number of ends in such a way that the boundary of every face is a cycle of $G$. Such an embedding is usually called a strong embedding. For each vertex $v \in V(G)$, we denote by $d(v)$ the degree of $v$ in $G$, and by $F(v)$ the set of all faces having $v$ as a vertex. We assume $d(v) \geq 3$ for all $v \in V(G)$ and every face of the embedding is homeomorphic to a closed disk. The combinatorial curvature $\Phi_{G}(v)$ of $G$ at a vertex $v$ is defined by

$$
\Phi_{G}(v):=1-\frac{d(v)}{2}+\sum_{\sigma \in F(v)} \frac{1}{\#(V(\bar{\sigma}))} .
$$

Conjecture 3.2 (Higuchi [1]). Let $G$ be a (possibly infinite) graph embedded in the plane such that every face has at least 3 sides. If $\Phi_{G}(v)>0$ for all $v \in V(G)$, then $G$ is a finite graph.

A polytopal 2-manifold with a finite number of ends is said to be regular if each polytopal 2-cell is a regular open polygon of unit side length. Clearly, the 1-skeleton of a regular polytopal 2-manifold can be considered as a simple graph embedded in a 2-manifold with a finite number of ends.

Conversely, let a simple graph $G$ be embedded in a 2-manifold $M$ with a finite number of ends. For each face $\sigma$ of the embedding, it is easy to find an embedding $\phi_{\sigma}: \bar{\sigma} \rightarrow \mathbb{R}^{2}$ such that $\phi_{\sigma}(\bar{\sigma})$ is a regular polygon of unit side length. So a 2 -cell embedding is the same as a polytopal 2-manifold with a finite number of ends. Notice that for each vertex $v \in V(G)$, the Gaussian curvature $\kappa_{M}(v)$ in this special case is the same as the combinatorial curvature $\Phi_{G}(v)$.

The combinatorial curvature is closely related to the continuous curvature of differential geometry. To obtain some finiteness results on discrete curvature, Chen and Chen [7] introduced absolute total curvature, and obtained the following relation between the finiteness of total curvature and the finiteness of the number of vertices of nonvanishing curvature. 
Theorem 3.3 (Chen and Chen [7]). Let $G$ be a (possibly infinite) graph embedded in a 2-manifold $M$ without boundary. If the absolute total curvature $|\Phi|(G)=$ $\sum_{v \in V(G)}\left|\Phi_{G}(v)\right|$ is finite, then the graph $G$ has only a finite number of vertices of nonvanishing curvature.

Higuchi's conjecture is modified by Chen and Chen [7] as follows.

Conjecture 3.4. Let $G$ be a (possibly infinite) graph embedded in a 2-manifold without boundary. If $\Phi_{G}(v) \geq 0$ for all $v \in V(G)$, then $G$ has a finite number of vertices of nonvanishing curvature.
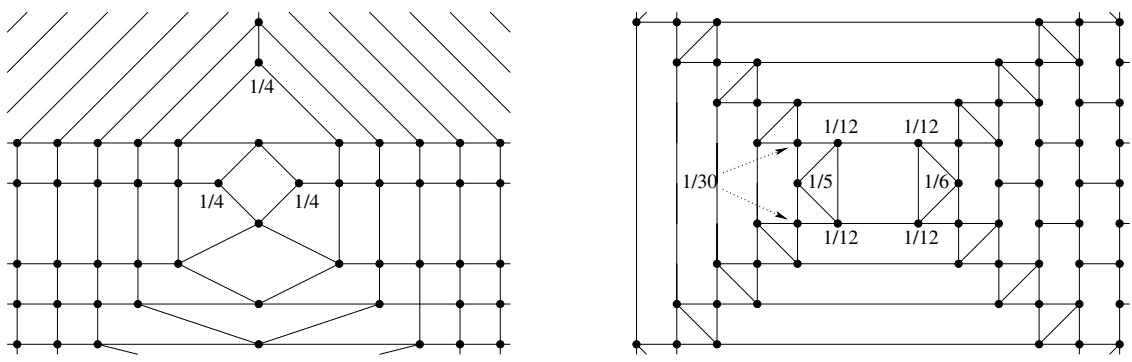

Figure 1. Infinite plane graphs with nonnegative curvature everywhere and positive curvature at a finite number of vertices.
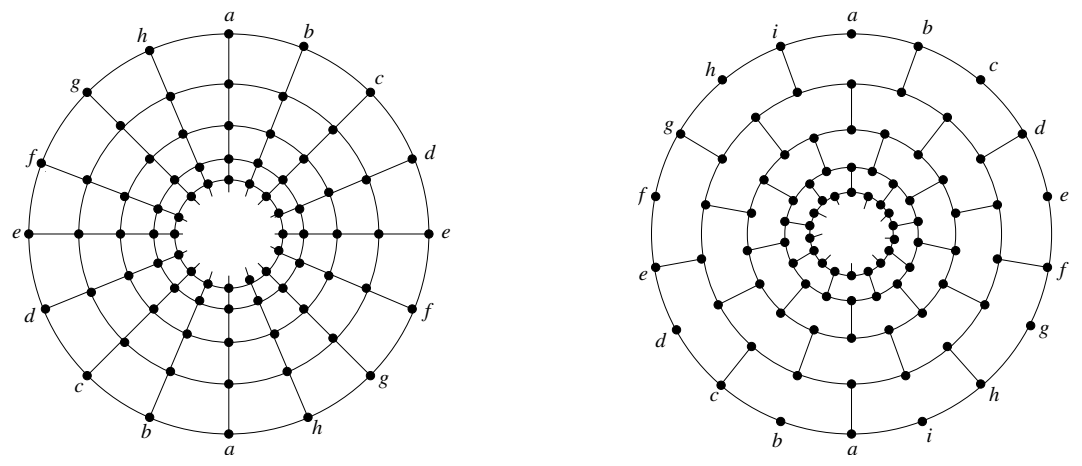

FiguRE 2. Infinite projective plane graphs with vanishing curvature everywhere.

In what follows we shall see that Theorems 3.1 and 3.3 imply the following characterization of regular polygonal surfaces with nonnegative curvature. The characterization solves Conjecture 3.4. However, the classification of embedded graphs having nonnegative combinatorial curvature remains open, and it seems complicated. Figure 1 shows two examples of infinite graphs embedded in the plane, having nonnegative combinatorial curvature everywhere and positive curvature at a finite number of vertices but at least one. Figure 2 shows two examples of infinite graphs embedded in the projective plane with vanishing curvature everywhere. Similar examples of infinite graphs embedded in the cylinder without boundary can be easily constructed to have vanishing curvature everywhere. 
Theorem 3.5. Let $G$ be a (possibly infinite) graph embedded in a 2-manifold $M$ without boundary such that every face has at least 3 sides. If $\Phi_{G}(v) \geq 0$ for all $v \in V(G)$, then the number of vertices with nonvanishing curvature is finite.

Furthermore, if $G$ is finite, then $M$ has four choices: sphere, torus, projective plane, and Klein bottle. If $G$ is infinite, then $M$ has three choices: plane, cylinder without boundary, and projective plane minus one point.

Proof. Since $\kappa_{M}(v)=\Phi_{G}(v) \geq 0$ for all $v \in V(M)$, it is obvious that

$$
\sum_{v \in V(M), \kappa_{M}(v)<0} \kappa_{M}(v)
$$

is zero; of course it is convergent. Note that $\nu(M)=1$ because each polygon is regular and has unit side length. Then by Theorem 3.1, the absolute total curvature is bounded above, i.e.,

$$
\sum_{v \in V(G)}\left|\Phi_{G}(v)\right|=\sum_{v \in V(M)} \kappa_{M}(v) \leq \chi(M) .
$$

Applying Theorem 3.3. we see that the graph $G$ has a finite number of vertices of nonvanishing curvature. Recall that when $N$ is a closed 2-manifold, the Euler characteristic of $N$ is given by

$$
\chi(N)= \begin{cases}2-2 g & \text { if } N \text { is orientable of genus } g \geq 0, \\ 2-g & \text { if } N \text { is nonorientable of genus } g \geq 1 .\end{cases}
$$

If $G$ is finite, then $M$ has no end, $M=N$ is a closed 2-manifold, and $\chi(M)=$ $\chi(N) \geq 0$ by the Gauss-Bonnet formula. Hence $\chi(N)=0,1,2$. So $M$ has four choices: sphere, torus, projective plane, and Klein bottle.

If $G$ is infinite, then $M$ has at least one end. Adding the ends to $M$, we obtain a closed 2-manifold $N$. Let $m$ be the number of ends of $M$. Then $\chi(N)=\chi(M)+m \geq$ $m$ by the Gauss-Bonnet formula. We have $\chi(N)=1,2$. So $M$ has three choices: plane, cylinder without boundary, and projective plane minus one point.

Theorem 3.6. Let $S$ be a cellular subspace of the polytopal d-manifold $M$. If the absolute total combinatorial curvature $|\Phi|(S)=\sum_{v \in V(M)}\left|\Phi_{S}(v)\right|$ is finite and if $\#(V(\bar{\sigma})) \leq m$ for all $d$-cells $\sigma$, then the number of vertices of nonvanishing combinatorial curvature for $S$ is bounded by $m !|\Phi|(S)$.

Proof. For each vertex $v \in V(M)$, let $f_{i j}(v)$ denote the number of $i$-cells $\sigma$ such that $\sigma \subset S, v \in \bar{\sigma}$, and $\#(V(\bar{\sigma}))=j$. Let $A$ be the $(d+1)$-by- $m$ matrix whose $(i, j)$-entry is $a_{i j}=\frac{(-1)^{i}}{j}$. Let $F_{v}$ be the $(d+1)$-by- $m$ matrix whose $(i, j)$-entry is $f_{i j}$. Then $\Phi_{X}(v)$ can be written as

$$
\Phi_{S}(v)=\left\langle A, F_{v}\right\rangle:=\sum_{i=0}^{d} \sum_{j=1}^{m} \frac{(-1)^{i} f_{i j}}{j} .
$$

Notice that the entries of the matrix $A$ are rational numbers and $\{B \mid\langle A, B\rangle=0\}$ is a hyperplane of the vector space of $(d+1)$-by- $m$ real matrices. It is easy to see that for any integral matrix $F$, if $\langle A, F\rangle \neq 0$, then $|\langle A, F\rangle| \geq \frac{1}{m !}$. Thus

$$
|\Phi|(S)=\sum_{v \in V(M), \Phi_{S}(v) \neq 0}\left|\Phi_{S}(v)\right| \geq \frac{\#\left\{v \in V(M) \mid \Phi_{S}(v) \neq 0\right\}}{m !} .
$$


Corollary 3.7. Let $S$ be a cellular subspace of a simplicial d-manifold $M$. If the absolute total combinatorial curvature $|\Phi|(S)=\sum_{v \in V(M)}\left|\Phi_{S}(v)\right|$ is finite, then the number of vertices of nonvanishing combinatorial curvature for $S$ is bounded by $d !|\Phi|(S)$.

Let $\mathrm{PS}_{n}$ ( $n$ is even) denote the infinite projective plane 4-regular graphs; see the example for $n=16$ in the left of Figure 2. Let $\mathrm{PH}_{n}$ ( $n$ is odd) denote the infinite projective plane 3-regular graphs; see the example for $n=9$ in the right of Figure2. The graphs $\mathrm{PS}_{n}$ and $\mathrm{PH}_{n}$ are actually the square and hexagon tessellations of the projective plane. Similar families of infinite graphs embedded in the cylinder $S^{1} \times(0,1)$ without boundary can be defined by tessellations; there are more families since there are two ends and cells can be wrapped around $S^{1}$ with shift. A question similar to the following can also be asked.

Question. Are the two families $\mathrm{PS}_{n}$ and $\mathrm{PH}_{n}$ the only infinite graphs embedded in the projective plane with nonnegative combinatorial curvature everywhere?

\section{ACKNOWLEDGMENT}

The author thanks the referee for reading the manuscript carefully and offering valuable comments.

\section{REFERENCES}

[1] A.D. Aleksandrov and V.A. Zalgaller, Intrinsic Geometry of Surfaces, Translations of Mathematical Monographs, vol. 15, Amer. Math. Soc., Providence, RI, 1967. MR0216434 (35:7267)

[2] C.B. Allendoerfer and A. Weil, The Gauss-Bonnet theorem for Riemannian polyhedra, Trans. Amer. Math. Soc. 53 (1943), 101-129. MR0007627 (4:169e)

[3] T. Banchoff, Critical points and curvature for embedded polyhedra, J. Diff. Geom. 1 (1967), 245-256. MR0225327 (37:921)

[4] J. Cheeger and D.G. Ebin, Comparison Theorems in Riemannian Geometry, North-Holland Publishing Co., 1975. MR0458335 (56:16538)

[5] J. Cheeger, W. Müller, and R. Schrader, On the curvature of piecewise flat spaces. Comm. Math. Phys. 92 (1984), 405-454. MR734226 (85m:53037)

[6] B. Chen, The Gram-Sommerville and Gauss-Bonnet theorems and combinatorial geometric measures for noncompact polyhedra, Advances in Math. 91 (1992), 269-291. MR1149626 (92m:52021)

[7] B. Chen and G. Chen, Gauss-Bonnet formula, finiteness condition, and characterizations of graphs embedded in surfaces, Graphs and Combin. 24 (2008), 159-183. MR2410938

[8] M. DeVos and B. Mohar, An analogue of the Descartes-Euler formula for infinite graphs and Higuchi's conjecture, Trans. Amer. Math. Soc. 359 (2007), 3287-3300. MR2299456 (2008e:05041)

[9] H. Groemer, On the extension of additive functionals on classes of convex sets, Pacific J. Math. 75 (1978), 397-410. MR0513905 (58:24003)

[10] M. Gromov, Hyperbolic groups, Essays in group theory, S. M. Gersten (Editor), M.S.R.I. Publ. 8, Springer, 1987, pp. 75-263. MR919829 (89e:20070)

[11] Y. Higuchi, Combinatorial curvature for planar graphs, J. Graph Theory 38 (2001), 220-229. MR:1864922 (2002i:05109)

[12] M. Ishida, Pseudo-curvature of a graph, Lecture at 'Workshop on topological graph theory', Yokohama National University, 1990.

[13] P. McMullen, Non-linear angle-sum relations for polyhedral cones and polytopes, Math. Proc. Cambridge Philos. Soc. 78 (1975), 247-261. MR0394436 (52:15238)

[14] S.B. Myers, Riemannian manifolds with positive mean curvature, Duke Math. J. 8 (1941), 401-404. MR0004518 (3:18f) 
[15] D. Stone, A combinatorial analogue of a theorem of Myers, Illinois J. Math. 20 (1976), 12-21. MR.0410602 (53:14350a)

[16] W. Woess, A note on tilings and strong isoperimetric inequality, Math. Proc. Camb. Phil. Soc. 124 (1998), 385-393. MR1636552 (99f:52026)

Department of Mathematics, Hong Kong University of Science and Technology, Clear Water Bay, Kowloon, Hong Kong

E-mail address: mabf chen@ust.hk 